\title{
Ion-Exchange-Resin-Supported Hydrogen Fluoride for Fluorination Reactions
}

\section{Key words}

hydrogen fluoride

fluorination

flow reaction

hydrofluorination

ion-exchange resin

Selected examples:
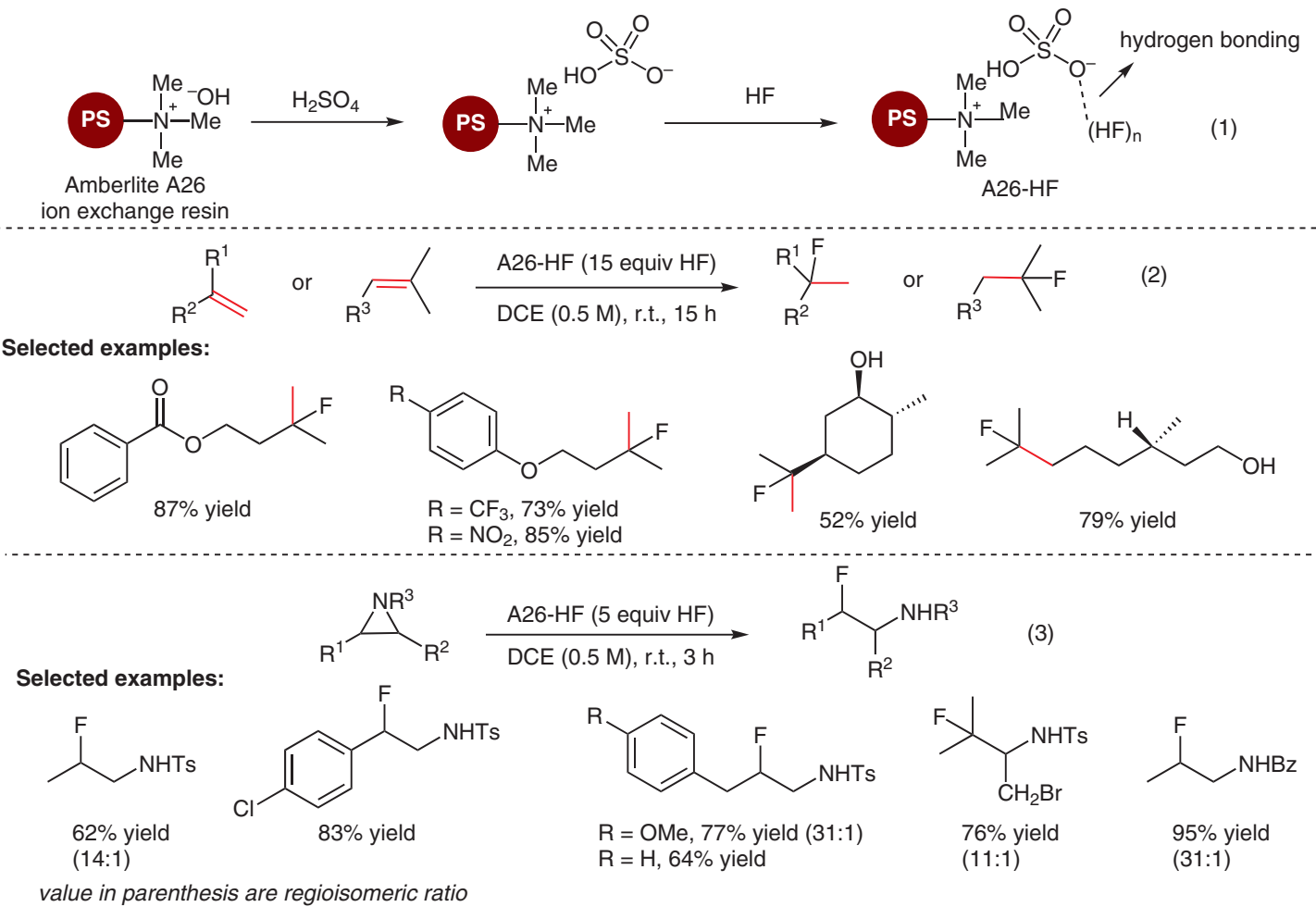

value in parenthesis are regioisomeric ratio

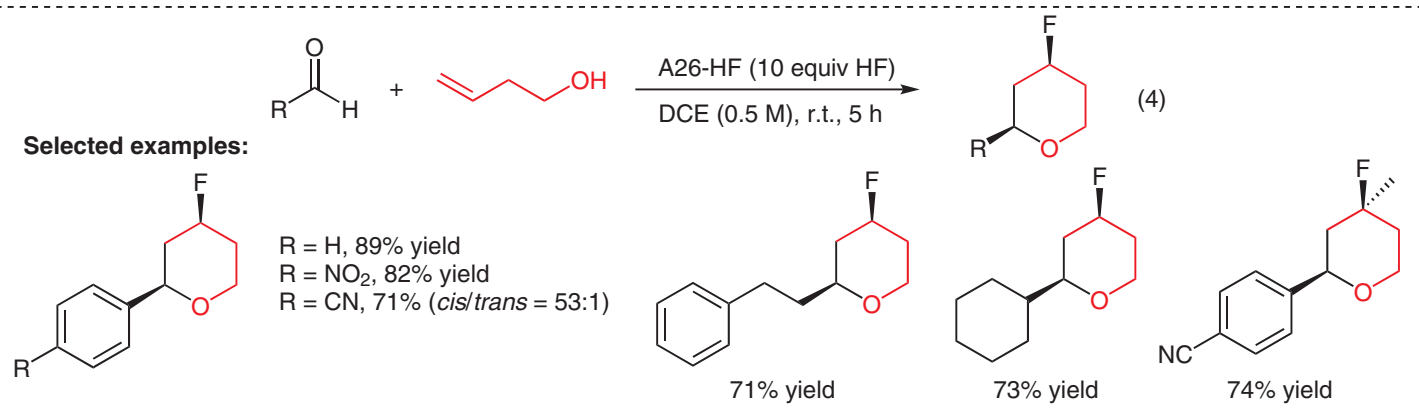

Significance: A solid anhydrous hydrogen fluoride-equivalent reagent (A26-HF) was prepared by treatment of ion-exchange resin with $\mathrm{HF}$ gas (eq. 1). A26-HF was used as a source of HF in the hydrofluorination of alkenes (eq. 2), the ring-opening fluorination of aziridines (eq. 3), and a fluoro-Prins reaction (eq. 4) to give a variety of fluorinated products in up to $89 \%$ yield.
Comment: The ion-exchange-resin-supported hydrogen fluoride was applied to the flow hydrofluorination of 3-methylbut-3-en-1-yl benzoate to give 3-fluoro-3-methylbutyl benzoate in 92\% NMR yield. In a thermostability test, only a $2 \mathrm{wt} \%$ loss of HF was detected when A26-HF was exposed to air at room temperature for 30 hours. 